\title{
Highly Nonlinear and Birefringent Spiral Photonic Crystal Fiber
}

\author{
S. Revathi, Srinivasa Rao Inbathini, and Rizwan Ali Saifudeen \\ School of Electronics and Communication, VIT University, Vellore, Tamil Nadu 632014, India \\ Correspondence should be addressed to S. Revathi; srevathi@vit.ac.in
}

Received 28 February 2014; Accepted 22 May 2014; Published 11 June 2014

Academic Editor: Jayanta K. Sahu

Copyright (C) 2014 S. Revathi et al. This is an open access article distributed under the Creative Commons Attribution License, which permits unrestricted use, distribution, and reproduction in any medium, provided the original work is properly cited.

\begin{abstract}
We propose and design a spiral photonic crystal fiber with elliptical air holes for achieving high birefringence, large nonlinearity, and negative dispersion. The structure is designed using chalcogenide glass $\left(\mathrm{As}_{2} \mathrm{~S}_{3}\right)$ for different ellipticity ratios of air holes in the cladding and the effect on various properties is observed. The proposed structure has birefringence of the order $10^{-2}$, nonlinearity of $26739.42 \mathrm{~W}^{-1} \mathrm{~m}^{-1}$, and dispersion of -1136.69 at $0.85 \mu \mathrm{m}$. An accurate numerical approach based on finite element method is used for the design and simulation of the structure. Due to high birefringence and negative dispersion, the proposed structure can be used for polarization control and dispersion compensation, respectively.
\end{abstract}

\section{Introduction}

Photonic crystal fiber is a class of optical fiber which is based on the properties of photonic crystal. Due to its unique properties photonic crystal fiber finds a lot of applications in optical fiber communication [1]. In standard fiber, the guiding mechanism is by a region with higher refractive index than the surrounding cladding. In case of PCFs, the microstructure of core and air holes surrounding it provide the required index difference. The basic principle of operation of a PCF is based either on modified total internal reflection in which light is guided in solid core which depends on the relative refractive index difference between the core and the microstructure cladding or on photonic band gap effect in which light is confined by a photonic band gap that is created by the microstructure cladding [2-4]. Such fiber allows the propagation of light through lower index core or even through hollow core. Compared to conventional fiber, PCFs provide large freedom in the design which allows the flexibility in various properties including birefringence, flattened and negative dispersion, effective area, and nonlinearity.

Apart from the conventional hexagonal lattice, different types of air hole arrangements are proposed for achieving many useful properties [5-7]. In equiangular spiral photonic crystal fiber, the air holes are distributed around the solid core so that the arrangement gives a spiral pattern. The design parameters for spiral PCF include number of spiral arms, number of air holes in each spiral arm, and core diameter. Various useful properties can be obtained by tailoring these parameters to the PCF design. Equiangular spiral PCF (ESPCF) with small effective area and large nonlinearity has been reported [8]. Spiral PCF with elliptical core is designed [9] which has large birefringence and nonlinearity. High birefringence in spiral PCF is obtained [10] by removing two identical air holes in the inner ring so as to make the design asymmetrical. The ES-PCF is much compact and simple as compared to conventional PCF. Spiral photonic crystal fibers can achieve better overlapping between the optical fields of the fundamental mode at a desired frequency compared to conventional PCF [11]. So it can be used for nonlinear applications like four-wave mixing, supercontinuum generation, and second harmonic generation.

\section{Properties of Photonic Crystal Fiber}

The various properties like birefringence, nonlinearity, and chromatic dispersion are discussed. Birefringence is a property of a material in which the refractive index depends on the direction of propagation of light and the polarization of the transmitted light. Birefringence is defined as the maximum difference in refractive index shown by the material. Consider

$$
B=\left|\operatorname{Re}\left(n_{\text {eff }}^{x}\right)-\operatorname{Re}\left(n_{\text {eff }}^{y}\right)\right|,
$$


where $n_{\mathrm{eff}}^{x}$ and $n_{\mathrm{eff}}^{y}$ are effective refractive indexes of the two fundamental polarized modes.

Birefringence in photonic crystal fibers can be obtained using asymmetric structures [12]. The fiber core can be designed to be asymmetrical by introducing double or triple defects in the fiber core [13]. High birefringence can be achieved by using double or triple core fiber $[14,15]$. The birefringence measured for this fiber at $1550 \mathrm{~nm}$ for fundamental mode was $2 \times 10^{-4}$. By introducing elliptical air holes in the cladding large birefringence can be achieved. High birefringence of the order of $10^{-2}$ is achieved in photonic crystal fiber with squeezed elliptical holes [16]. The birefringence varies with the variation of the normalized frequency, size ratio, and effective area of the elliptical air holes. Asymmetrical structures can be used to achieve large birefringence by spiral arrangement of air holes in the cladding or by introducing an elliptical core [17].

Dispersion occurs in all types of fibers and is a result of finite spectral line width of optical source. Chromatic dispersion is the variation in group velocity with a variation in wavelength. This variation in velocity results in broadening of pulses when traveling through the fiber, which overlap and result in increased bit error rate. Increase in bit error rate affects the signal quality. In high speed transmission, chromatic dispersion is a major limiting factor. Chromatic dispersion includes material dispersion and wave guide dispersion. The chromatic dispersion in PCF is given by

$$
D=-\frac{\lambda}{c} \frac{d^{2} n_{\mathrm{eff}}}{d \lambda^{2}} \mathrm{ps} \mathrm{nm}^{-1} \mathrm{~km}^{-1}
$$

where $c$ is the velocity of light and $\lambda$ is the operating wavelength. The refractive index corresponding to the operating wavelength is given by Sellemier's formula:

$$
n^{2}-1=\sum_{i}\left(\frac{A_{i} \lambda^{2}}{\lambda^{2}-\lambda_{1}^{2}}\right)
$$

where $A$ is the Sellmeier coefficient.

Chromatic dispersion in PCF can be adjusted by various methods [18], either by changing the arrangement of air holes or with the number of air holes, and also with the shape of the air holes in the cladding region. Properly designed PCFs with large negative dispersion coefficient value can be used for dispersion compensation $[19,20]$. There is large wave guide contribution to material dispersion in index guiding PCFs with small core, so anomalous dispersion can be attained. Such fibers can be used for dispersion management in visible wavelength with suitable cladding design by varying the pitch and size of the air holes. by

The nonlinearity in a photonic crystal fiber is calculated

$$
\gamma=\frac{2 \pi n_{2}}{\lambda A_{\text {eff }}} \mathrm{W}^{-1} \mathrm{~m}^{-1}
$$

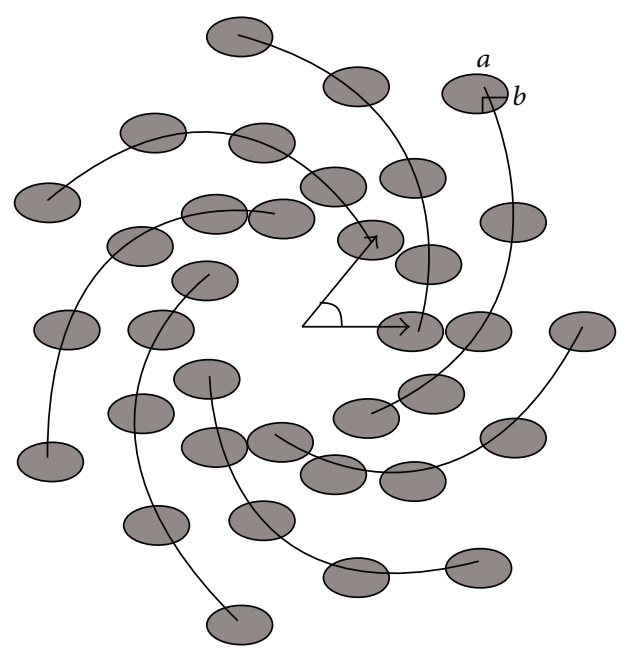

FIgURE 1: Structure of spiral PCF with elliptical air holes.

$A_{\text {eff }}$ is the effective area of the fundamental mode and $n_{2}$ is nonlinear refractive index (for $\mathrm{As}_{2} \mathrm{~S}_{3}, n_{2}=3.825 \times$ $10^{-18} \mathrm{~m}^{2} / \mathrm{w}$ ). Effective area is expressed as

$$
A_{\mathrm{eff}}=\frac{\left[\iint|E(x, y) d x d y|^{2}\right]^{2}}{\iint|E(x, y) d x d y|^{4}} \mu \mathrm{m}^{2} .
$$

Enhanced nonlinearity can be achieved by the use of different materials and different structures of PCF [21, 22]. In photonic crystal fiber nonlinearities come into action, when the effective mode area of propagation of light is very small. Nonlinearity depends on the nonlinear refractive index of the material. The larger the effective mode area is, the smaller the nonlinearity value is. Certain materials like soft-glass and chalcogenide exhibit large value of nonlinearity [23, 24]. Effective area in a PCF is the area where light is confined in a fiber. In PCFs, the effective area includes the core region and a small fraction of areas in the cladding region. $A_{\text {eff }}$ depends on various fiber parameters like the core radius and the corecladding index difference. The effective area increases with the increase in diameter of air holes in the cladding region in spiral PCF [25].

\section{Structure and Design of Proposed Spiral PCF}

The structure is designed using the tool Comsol 3.5. It is based on FEM (finite element method), an accurate numerical method used to find solution to boundary value problems. The designed structure of a photonic crystal fiber with elliptical air holes in the cladding region is shown in the Figure 1. The structure has a spiral lattice of elliptical air holes. For attaining large value of birefringence elliptical air holes are included in the structure. The proposed structure consists of seven spiral arms, each with five elliptical air holes. Here, $a$ and $b$ represent the semimajor and semiminor axis of the elliptical air holes.

The angular displacement between two adjacent elliptical air holes in a ring is $180 / N$ degrees. The maximum value 


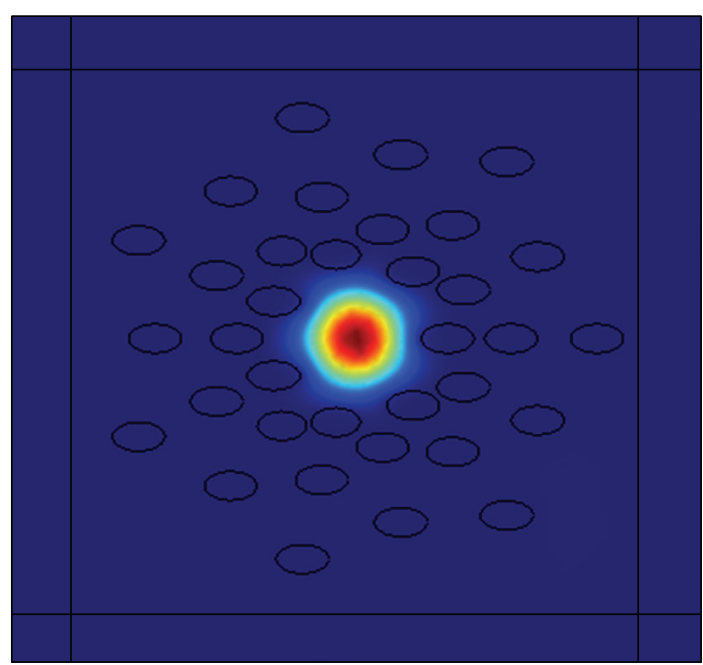

(a)

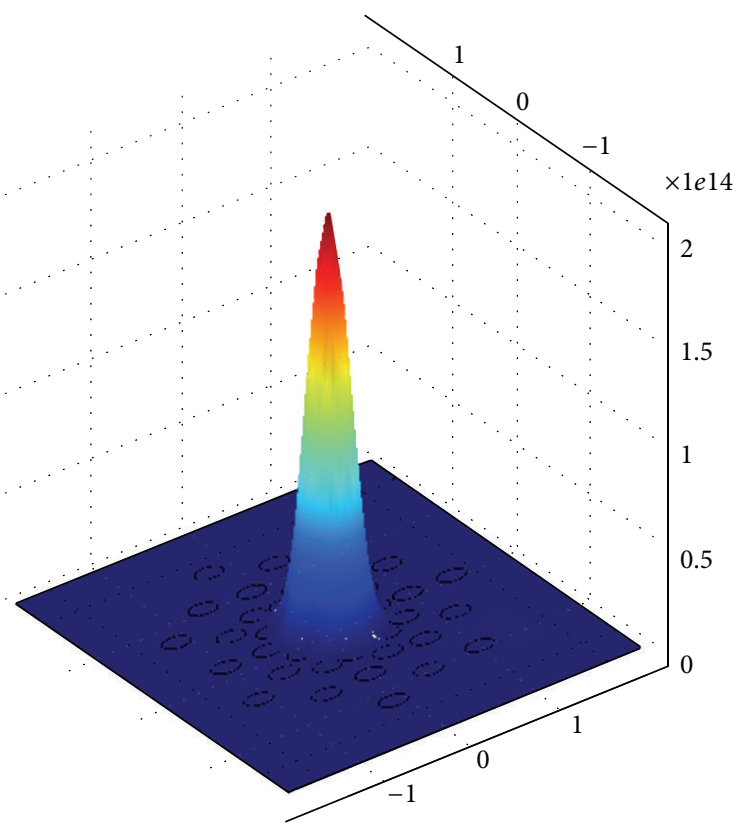

(b)

FIGURE 2: Simulated structure showing confinement of light (a) two-dimensional plot and (b) three-dimensional plot.

on $N$ or the number of air holes in a ring is limited by the core diameter and the size of the air holes. The distance between two subsequent air holes in each spiral arm follows geometric progression. The core diameter is approximately $0.7 \mu \mathrm{m}$. The width of the PML layer is fixed to be $1 \mu \mathrm{m}$. The semimajor axis of the ellipse is $1.5 \mu \mathrm{m}$ and the semiminor axis is tailored to obtain different ellipticity ratios. The structure is designed for different ellipticity ratios of 1.4, 1.5, 1.6, and 1.7 for chalcogenide glass. The effect of ellipticity ratio on various fiber properties is observed. The spiral arrangement of elliptical air holes provides high birefringence. The advantage with spiral structure is that it is much compact and have small effective mode area, hence achieving large value for nonlinear coefficient. The smallest air hole size in the proposed structure is $3 \mu \mathrm{m}$ (major axis of elliptical air holes) which is much larger than the dimension in [26]; the proposed design can be fabricated using the stack and draw method.

We can calculate the effective modal index $\left(n_{\text {eff }}\right)$ and effective area of the designed spiral photonic crystal fiber by using COMSOL 3.5, which is a finite element method based software. The spiral structure with elliptical air holes is distributed in chalcogenide glass $\left(\mathrm{As}_{2} \mathrm{~S}_{3}\right)$ with refractive index of 2.45.

Figure 2 shows the confinement of light in the fiber core at $1.55 \mu \mathrm{m}$ in chalcogenide glass spiral PCF.

\section{Results and Discussion}

A wide range of wavelength from $0.85 \mu \mathrm{m}$ to $2.45 \mu \mathrm{m}$ is varied for the analysis. The semimajor axis of the elliptic air holes is kept constant, and the semiminor axis is varied to obtain ellipticity ratios of $1.4,1.5,1.6$, and 1.7. The semiminor axis is $1.0714 \mu \mathrm{m}, 1 \mu \mathrm{m}, 0.9375 \mu \mathrm{m}$, and $0.8823 \mu \mathrm{m}$ for ellipticity ratios 1.4, 1.5, 1.6, and 1.7. The variation of effective refractive index $\left(n_{\text {eff }}\right)$ with wavelength is shown in Figure 3, which depicts $n_{\text {eff }}$ decreases with the increase in wavelength. It is found that with the increase in ellipticity ratio of the air holes in the cladding the $n_{\text {eff }}$ decreases.

In contrast to the conventional fiber, the spiral PCF provides more freedom in tailoring birefringence. Here we examine the dependence of birefringence on ellipticity ratio of air holes in the cladding region for different wavelength range. In Figure 4 we observe that with the increase in wavelength the birefringence increases.

Birefringence in the order of $10^{-2}$ is achieved. Figure 5 shows the effect of ellipticity of air holes on birefringence. The birefringence obtained is better than that with conventional elliptical air holes [12, 17]. It is observed that there is an increase in birefringence with the increase in ellipticity ratio of the air holes. For ellipticity ratios of 1.4, 1.5, and 1.6 the birefringence is of the order of $0.014,0.02$, and 0.0225 , respectively, at $1.55 \mu \mathrm{m}$. Birefringence of 0.0256 is obtained for ellipticity ratio of 1.7 at $1.55 \mu \mathrm{m}$ better than that reported in [17]. The more the increase in ellipticity ratio is, the more asymmetrical the structure becomes. When the effective area in PCF cladding increases, there exists a difference in the air filling fraction between the $x$ and $y$ directions. As a result there is more confined field on one of the directions either $x$ or $y$ depending on the structure of the air holes.

Dispersion in a PCF is highly influenced by the effect of size and positioning of the air holes in the cladding region. Figure 6 shows the variation of chromatic dispersion with wavelength. Material dispersion is included. It has been observed that the dispersion value is negative in the optical wavelength region for different ellipticity ratios. For ellipticity 


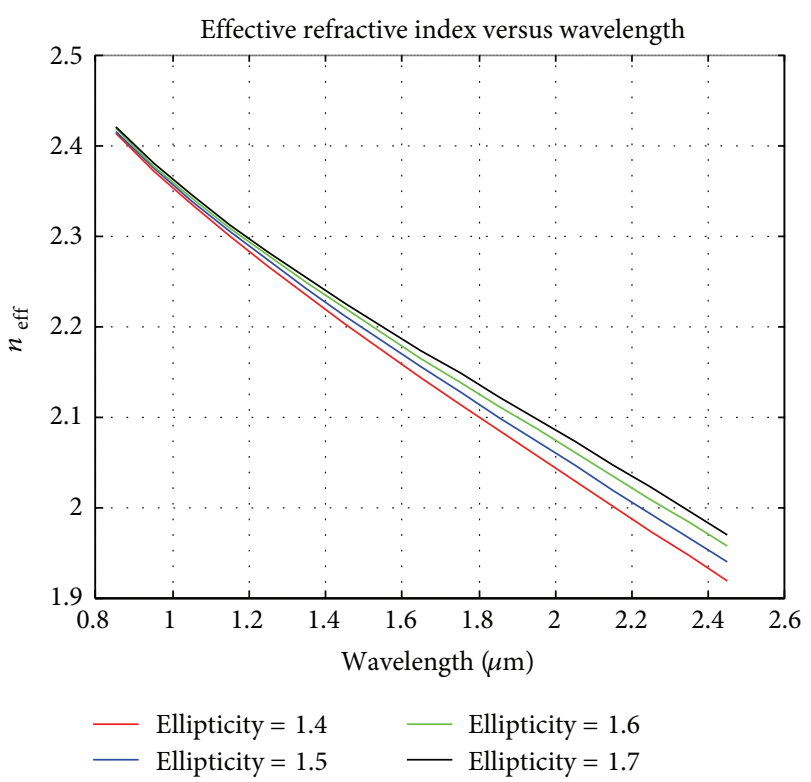

FIGURE 3: $n_{\text {eff }}$ versus wavelength for fixed ellipticity ratios of 1.4, 1.5, 1.6, and 1.7.

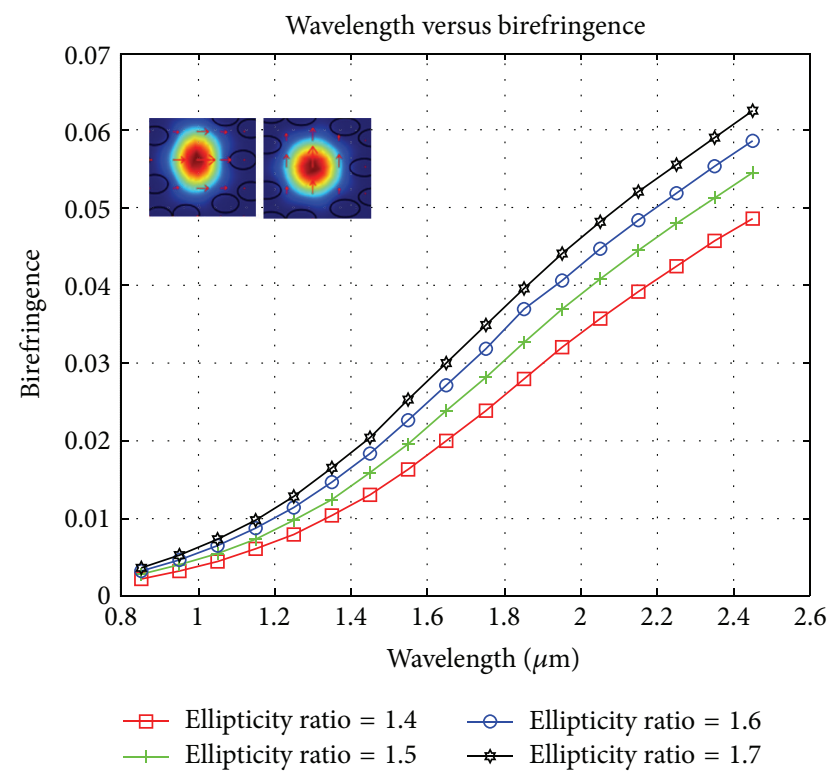

FIGURE 4: Birefringence versus wavelength for fixed ellipticity ratios of 1.4, 1.5, 1.6, and 1.7 with fundamental $X$ and $Y$ polarization modes.

ratio of 1.7 , the dispersion is $-1136.69 \mathrm{ps} / \mathrm{nm}-\mathrm{km}$ at $0.85 \mu \mathrm{m}$ and $-907.6 \mathrm{ps} / \mathrm{nm}-\mathrm{km}$ at $1.1 \mu \mathrm{m}$. At $1.55 \mu \mathrm{m}$ for ellipticity ratio of 1.4 , a dispersion of $-338 \mathrm{ps} / \mathrm{nm}-\mathrm{km}$ is obtained.

The structure offers two zero dispersions, one at $1.93 \mu \mathrm{m}$ and $2.21 \mu \mathrm{m}$ for ellipticity ratio of 1.6 and the other at $1.86 \mu \mathrm{m}$ and $2.25 \mu \mathrm{m}$ for ellipticity ratio of 1.7. For ellipticity ratio of 1.6 wavelength between $1.93 \mu \mathrm{m}$ and $2.21 \mu \mathrm{m}$ the structure offers a flattened dispersion with maximum value of $25.2 \mathrm{ps} / \mathrm{nm}$ $\mathrm{km}$. The negative dispersion of PCF can be used for dispersion compensation. Due to large value of negative dispersion as compared to conventional fiber, short length of PCF can be

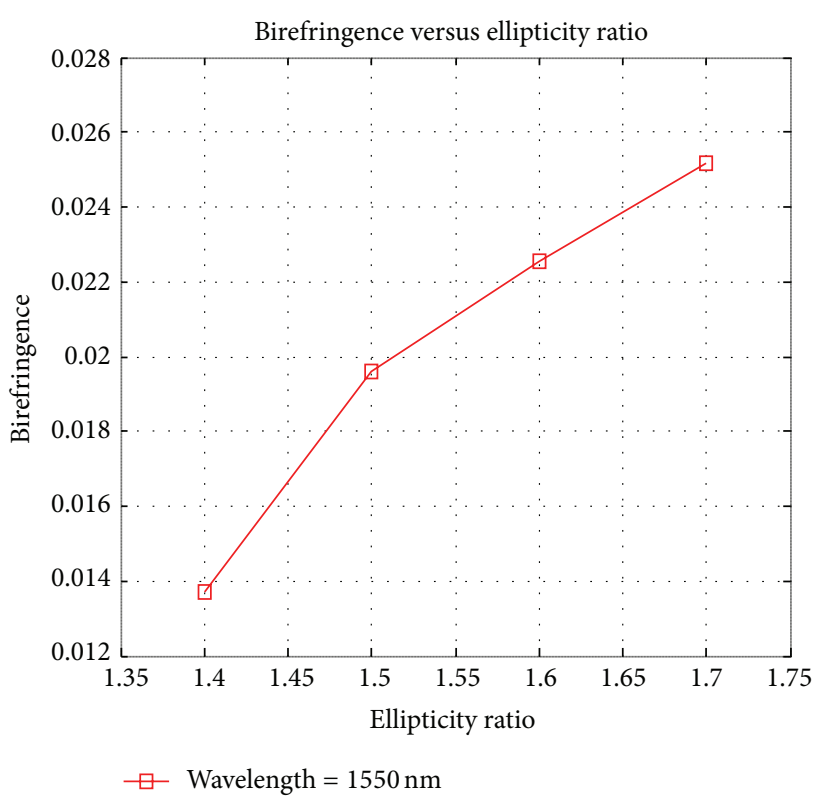

FIGURE 5: Birefringence versus ellipticity of air holes at wavelength of $1.55 \mu \mathrm{m}$.

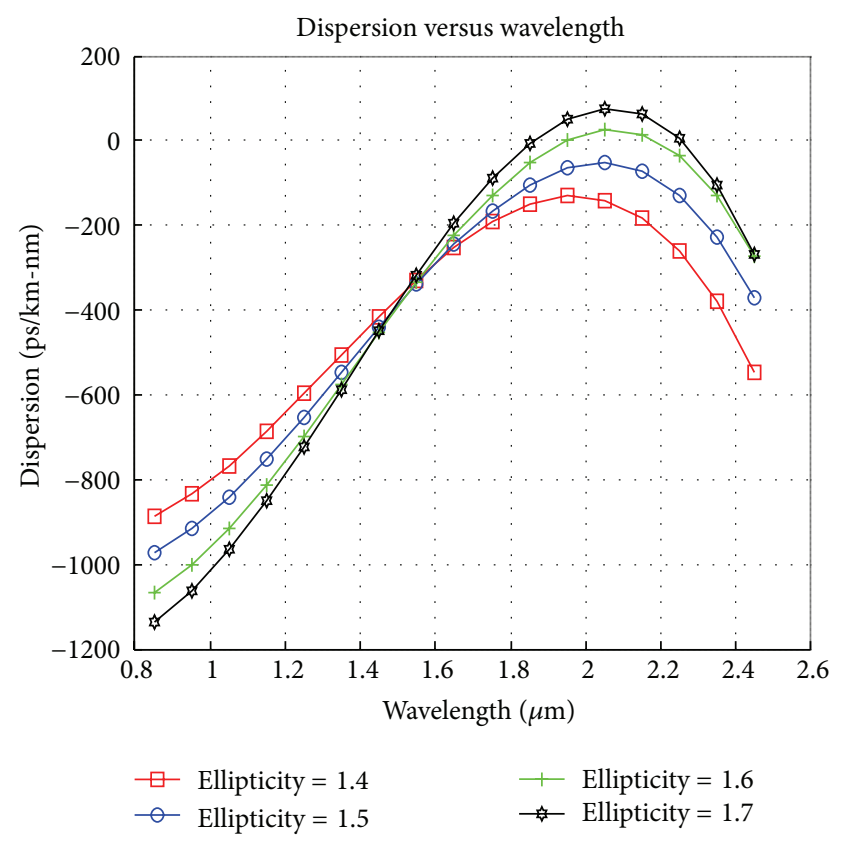

FIGURE 6: Dispersion versus wavelength for ellipticity ratios of 1.4, $1.5,1.6$, and 1.7.

used for dispersion compensation, thus reducing the loss [27]. For ellipticity ratio of 1.5 the value of dispersion is $-338 \mathrm{ps} / \mathrm{nm}-\mathrm{km}$ for PCF.

For single mode fiber the typical value of chromatic dispersion is $17 \mathrm{ps} / \mathrm{nm}-\mathrm{km}$. The length of the dispersion compensating fiber is given by [28]

$$
L_{\mathrm{DCF}}=\frac{L_{\mathrm{SMF}} \times D_{\mathrm{SMF}}}{D_{\mathrm{DCF}}} .
$$




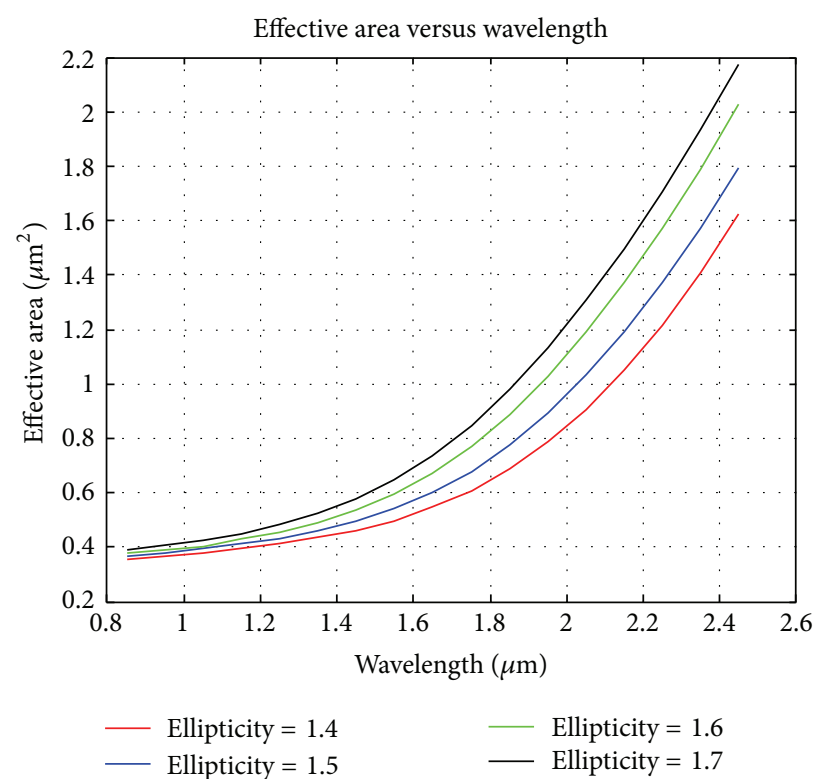

(a)



(b)

FIGURE 7: (a) Effective area versus wavelength. (b) Nonlinearity versus wavelength for ellipticity ratios 1.4, 1.5, 1.6, and 1.7.

For a span length of $80 \mathrm{Km}$ of single mode fiber the length of the dispersion compensating PCF is approximately $4 \mathrm{Km}$, which is small as compared to dispersion compensating single mode fiber. At the short wave IR region, due to flattened dispersion the fiber can be used in nonlinear application like supercontinuum generation.

Figure 7 shows the effect of ellipticity ratio on effective area and nonlinearity for wavelength range between $0.85 \mu \mathrm{m}$ to $2.5 \mu \mathrm{m}$. The effective area is very small in the order $10^{-13} \mathrm{~m}$ for the proposed structure. The effective area increases with ellipticity ratio. At $1.55 \mu \mathrm{m}$ the $A_{\text {eff }}$ is calculated to be $0.49 \mu \mathrm{m}^{2}, 0.54 \mu \mathrm{m}^{2}, 0.59 \mu \mathrm{m}^{2}$, and $0.64 \mu \mathrm{m}^{2}$ for ellipticity ratios of 1.4, 1.5, 1.6, and 1.7, respectively. The value is very smaller than that in conventional fiber. The structure shows very large nonlinearity as effective area is small and is inversely proportional to nonlinearity. The very high nonlinearity is because of large nonlinear refractive index of chalcogenide. The nonlinearity decreases as the wavelength increases because of reduced confinement of light in the core. The higher the confinement of light in the core region the lower the value of the effective area, and so high nonlinearity can be achieved. Large nonlinearity of $26739.42 \mathrm{~W}^{-1} \mathrm{~m}^{-1}$ is attained at $1.55 \mu \mathrm{m}$ which decreases with ellipticity ratio of the air holes. The obtained value of nonlinearity is better than that in $[20,29]$.

Thus our design is important (relative to others) because spiral PCF with soft glass reported [9] has a little higher birefringence, compared to our result, but the nonlinearity reported in our case is large and flattened and low dispersion is obtained and this combination is an added advantage to go for nonlinear application like supercontinuum generation.

\section{Conclusion}

A photonic crystal fiber with elliptical air holes in spiral lattice is designed by finite element method. The structure is designed for different ellipticity ratios for the air holes in the cladding and the effect on various fiber parameters is analyzed. From the numerical results, large birefringence of the order of $10^{-2}$ is obtained, which is larger than that of conventional PCFs. The fiber offers large birefringence value of 0.0256 at $1.55 \mu \mathrm{m}$. So, it finds application in polarization control. Due to the small core it offers large value for nonlinearity of $26739.42 \mathrm{~W}^{-1} \mathrm{~m}^{-1}$ at $1.55 \mu \mathrm{m}$ and a negative dispersion of -1136.69 at $0.85 \mu \mathrm{m}$, so that it can be used as dispersion compensation fiber. Compared to other ellipticity ratios, the structure with ellipticity ratio 1.6 is optimized and has better properties in terms of birefringence, dispersion, and nonlinearity. So it is evident that, large value of nonlinearity and flattened dispersion around short IR wave region leads this spiral PCF to be used in non-linear optical applications.

\section{Conflict of Interests}

The authors declare that there is no conflict of interests regarding the publication of this paper.

\section{References}

[1] P. S. J. Russell, "Photonic-crystal fibers," Journal of Lightwave Technology, vol. 24, no. 12, pp. 4729-4749, 2006.

[2] J. A. West, N. Venkataramam, C. M. Smith, and M. T. Gallagher, "Photonic crystal fibers," in Proceedings 27th European Conference on Optical Communication, Amsterdam, The Netherlands, 2002. 
[3] J. Broeng, T. Søndergaard, S. E. Barkou, P. M. Barbeito, and A. Bjarklev, "Waveguidance by the photonic bandgap effect in optical fibres," Journal of Optics A: Pure and Applied Optics, vol. 1, no. 4, pp. 477-482, 1999.

[4] J. Wang, C. Jiang, W. Hu, and M. Gao, "High birefringence photonic bandgap fiber with elliptical air holes," Optical Fiber Technology, vol. 12, no. 3, pp. 265-267, 2006.

[5] I. Kujawa, R. Buczynski, T. Martynkien et al., "Multiple defect core photonic crystal fiber with high birefringence induced by squeezed lattice with elliptical holes in soft glass," Optical Fiber Technology, vol. 18, no. 4, pp. 220-225, 2012.

[6] S. M. A. Razzak and Y. Namihira, "Proposal for highly nonlinear dispersion flattened octagonal photonic crystal fibers," Photonics Technology Letters, vol. 20, no. 4, pp. 249-251, 2008.

[7] A. Agrawal, N. Kejalakshmy, B. M. A. Rahman, and K. T. V. Grattan, "Soft glass equiangular spiral photonic crystal fiber for supercontinuum generation," IEEE Photonics Technology Letters, vol. 21, no. 22, pp. 1722-1724, 2009.

[8] M. N. Hossain, M. S. Alam, D. M. N. Hasan, and K. M. Mohsin, "A highly nonlinear spiral photonic crystal fiber for tailoring two zero dispersion wavelengths in the visible region," Photonics Letters of Poland, vol. 2, no. 3, pp. 143-145, 2010.

[9] C. Gui and J. Wang, "Elliptical-spiral photonic crystal fibers with wideband high birefringence, large nonlinearity, and low dispersion," IEEE Photonics Journal, vol. 4, no. 6, pp. 2152-2158, 2012.

[10] M. Samiul Habib, M. Selim Habib, M. Imran Hasan, and S. M. A. Razzak, "Highly nonlinear polarization maintaining two zero dispersion spiral photonic crystal fiber using artificial defects," Optical Fiber Technology, vol. 19, no. 6, pp. 539-542, 2013.

[11] I. N. M. Wijeratne, N. Kejalakshmy, A. Agrawal, B. M. A. Rahman, and K. T. V. Grattan, "Numerical analysis of second harmonic generation in soft glass equiangular spiral photonic crystal fibers," IEEE Photonics Journal, vol. 4, no. 2, pp. 357-368, 2012.

[12] J. Wang, C. Jiang, W. Hu, and M. Gao, "High birefringence photonic bandgap fiber with elliptical air holes," Optical Fiber Technology, vol. 12, no. 3, pp. 265-267, 2006.

[13] G. Hu and D. Chen, "Side-hole dual-core photonic crystal fiber for hydrostatic pressure sensing," Journal of Lightwave Technology, vol. 30, no. 14, pp. 2382-2387, 2012.

[14] Y. Du, X. Qiao, and Q. Rong, "A miniature fabry-pérot interferometer for high temperature measurement using a double-core photonic crystal fiber," IEEE Sensors Journal, vol. 14, no. 4, pp. 1069-1073, 2014.

[15] A. G. Coelho Jr., M. B. C. Costa, A. C. Ferreira, M. G. Da Silva, M. L. Lyra, and A. S. B. Sombra, "Realization of all-optical logic gates in a triangular triple-core photonic crystal fiber," Journal of Lightwave Technology, vol. 31, no. 5, pp. 731-739, 2013.

[16] P. Song, Y. Tong, and X. Chen, "A novel high birefringence photonic crystal fiber with squeezed elliptical holes," in Proceedings of the 3rd International Conference on BioMedical Engineering and Informatics (BMEI '10), pp. 3024-3027, October 2010.

[17] M. A. Islam and M. S. Alam, "Design of a polarization-maintaining equiangular spiral photonic crystal fiber for residual dispersion compensation over $\mathrm{E}+\mathrm{S}+\mathrm{C}+\mathrm{L}+\mathrm{U}$ wavelength bands," IEEE Photonics Technology Letters, vol. 24, no. 11, pp. 930-932, 2012.

[18] S. Gowre, S. Mahapatra, S. K. Varshney, and P. K. Sahu, "Dispersion characteristics of all-glass photonic crystal fibers," Optik, vol. 124, no. 18, pp. 3730-3733, 2013.
[19] G. N. Malheiros-Silveira, J. A. Mores Jr., and H. E. HernándezFigueroa, "Improving dispersion compensation of photonic crystal fibers through air hole core insertion," in Proceedings of the SBMO/IEEE MTT-S International Microwave and Optoelectronics Conference (IMOC '11), pp. 121-124, November 2011.

[20] B.-L. Wang, W.-H. Zheng, R.-J. Chen, and H.-K. Wei, "Dispersion compensation photonic crystal fibers with ultra-high dispersion coefficient and extremely low loss," in Proceedings of the 6th International Conference on Advanced Infocomm Technology (ICAIT '13), pp. 97-98, 2013.

[21] C. W. Rudy, A. Marandi, K. L. Vodopyanov, and R. L. Byer, "Octave-spanning supercontinuum generation in in situ tapered As 2S3 fiber pumped by a thulium-doped fiber laser," Optics Letters, vol. 38, no. 15, pp. 2865-2868, 2013.

[22] J. S. Sanghera, L. B. Shaw, C. M. Florea et al., "Non-linearity in chalcogenide glasses and fibers, and their applications," in Proceedings of the Quantum Electronics and Laser Science Conference (QELS '08), May 2008.

[23] B. Dabas and R. K. Sinha, "Dispersion characteristic of hexagonal and square lattice chalcogenide As2Se3 glass photonic crystal fiber," Optics Communications, vol. 283, no. 7, pp. 13311337, 2010.

[24] A. Agrawal, N. Kejalakshmy, B. M. A. Rahman, and K. T. V. Grattan, "Soft glass spiral PCF with flat anomalous dispersion at $1064 \mathrm{~nm}$ and $\mathrm{Y} \gg 2000 \mathrm{~W}^{-1} \mathrm{~km}^{-1}$ at $1550 \mathrm{~nm}$," in Proceedings of the Conference on Optical Fiber Communication (OFC '09), March 2009.

[25] R. K. Sharma, A. Mittal, and V. Agrawal, "A design of hybrid elliptical air hole ring chalcogenide As2Se3 glass PCF: application to lower zero dispersion," International Journal of Engineering Research and Technology, vol. 1, no. 3, 2012.

[26] D. C. Tee, M. H. Abu Bakar, N. Tamchek, and F. R. Mahamd Adikan, "Photonic crystal fiber in photonic crystal fiber for residual dispersion compensation over $\mathrm{E}+\mathrm{S}+\mathrm{C}+\mathrm{L}+\mathrm{U}$ wavelength bands," IEEE Photonics Journal, vol. 5, no. 3, 2013.

[27] S. Gowre, S. Mahapatra, S. K. Varshney, and P. K. Sahu, "Dispersion characteristics of all-glass photonic crystal fibers," Optik, vol. 124, no. 18, pp. 3730-3733, 2013.

[28] F. Gérôme, J.-L. Auguste, and J.-M. Blondy, "Design of dispersion-compensating fibers based on a dual-concentriccore photonic crystal fiber," Optics Letters, vol. 29, no. 23, pp. 2725-2727, 2004.

[29] F. Begum, Y. Zhang, S. Kaijage, Y. Namihira, and N. Zou, "Highly nonlinear photonic crystal fibers for optical coherence tomography applications," in Optical Sensors and Biophotonics, Proceedings of SPIE, November 2009. 

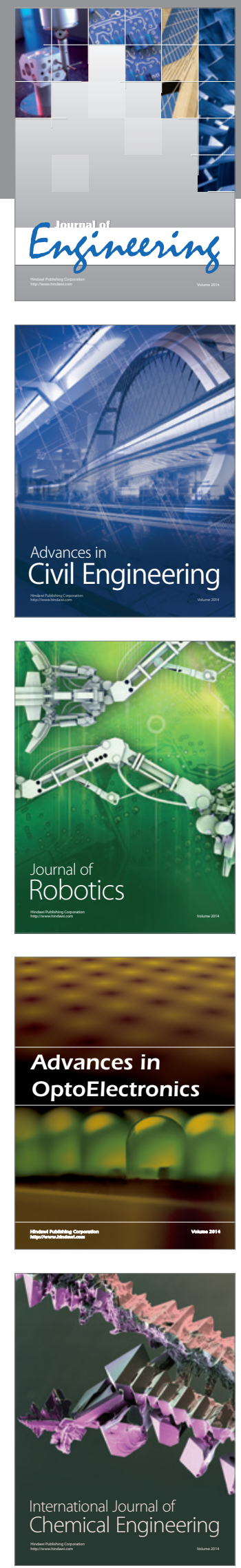

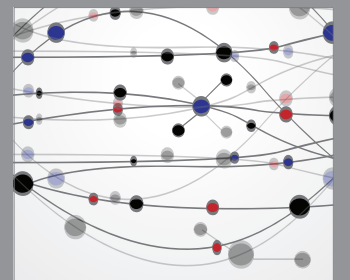

The Scientific World Journal
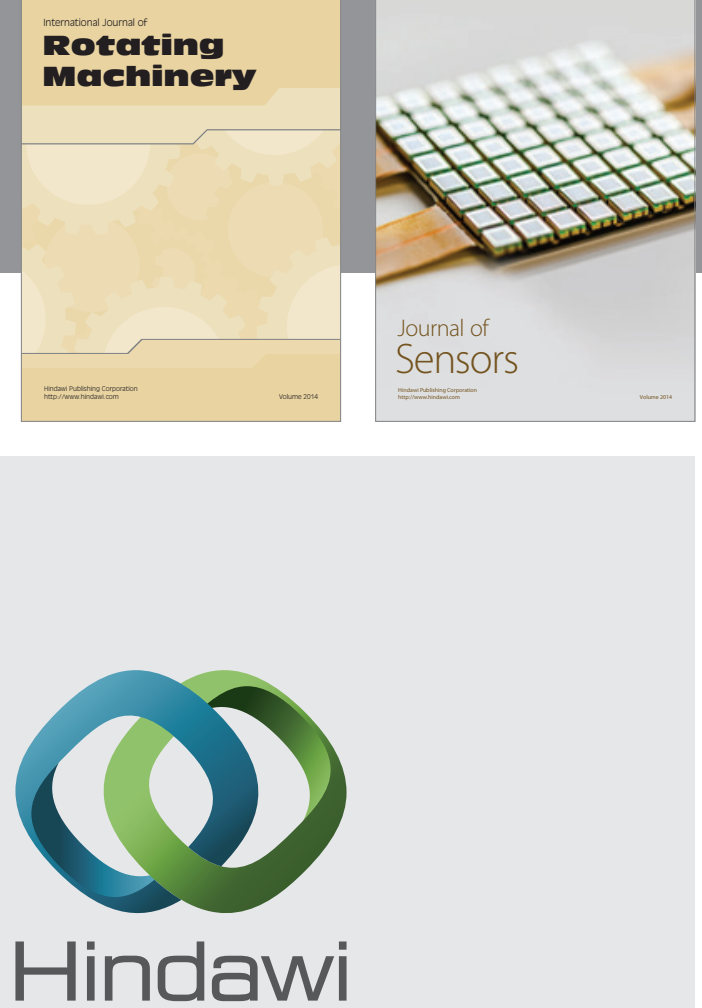

Submit your manuscripts at http://www.hindawi.com
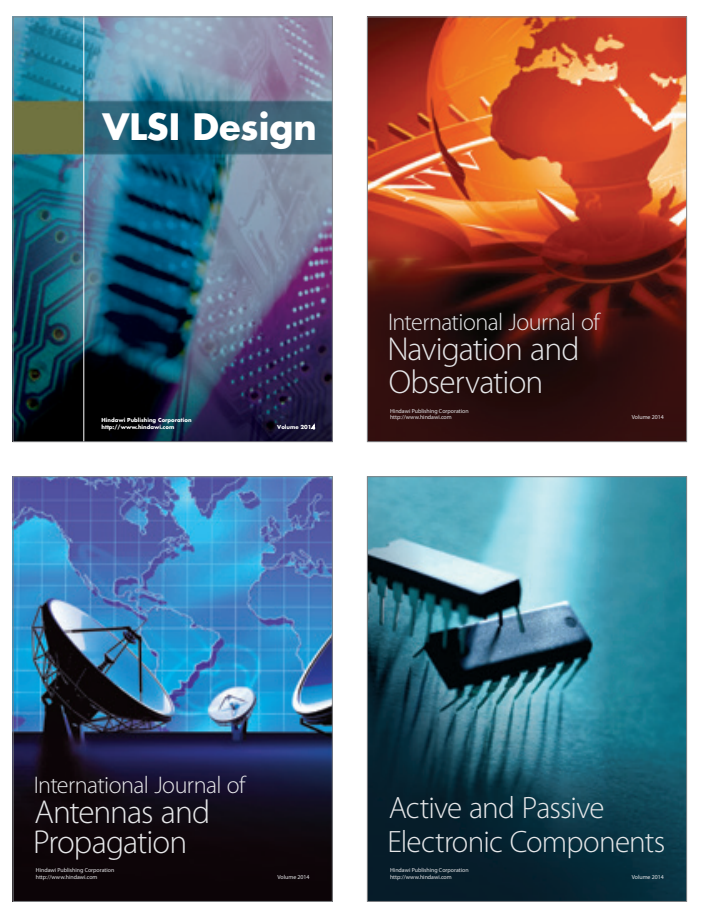
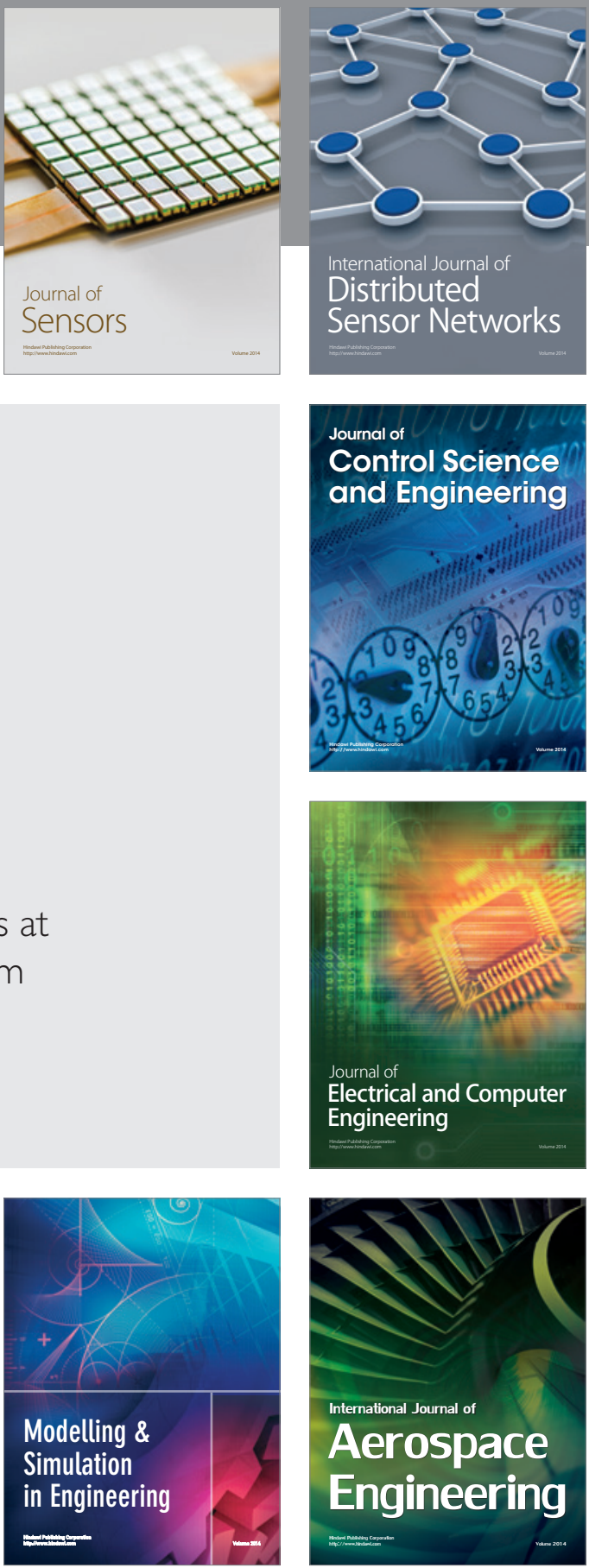

Journal of

Control Science

and Engineering
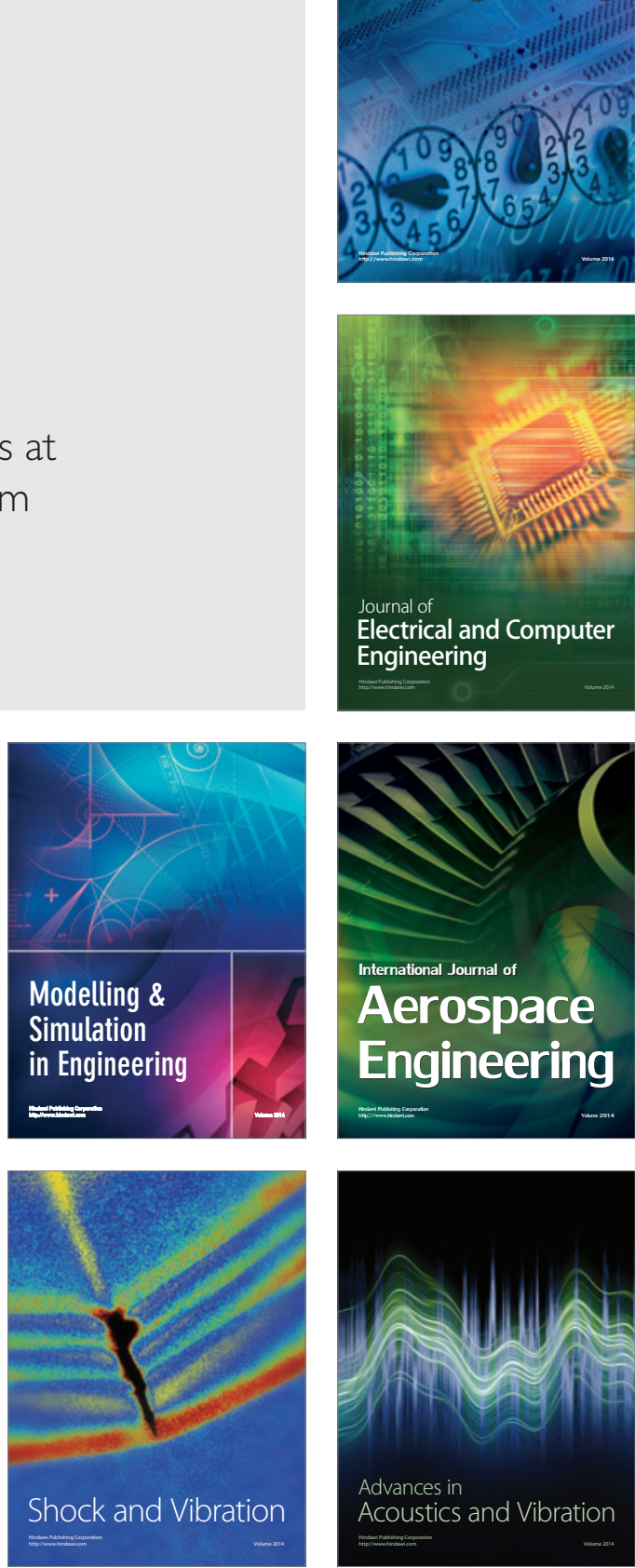